\title{
UNUSUAL PHOTOLUMINESCENCE OF POROUS CdS (CdSe) CRYSTALS.
}

\author{
R. Tenne and V.M. Nabutovsky, \\ Department of Materials Research, Weizmann Institute of Science Rehovot, 76100, ISRAEL. \\ E. Lifshitz and A.F. Francis, \\ Department of Chemistry, University of Michigan, Ann-Arbor, MI 48109-1055, USA
}

(Received 16 October 1991 by S. Alexander)

\begin{abstract}
Low temperature photoluminescence $(\mathrm{PL})$ is used to study photoetched $(\mathrm{PE}) \mathrm{CdS}$ (CdSe). This surface treatment produces a porous fractal-type morphology, with superior photovoltaic properties. While most of the usual features in the PL spectrum are suppressed after $\mathrm{PE}$, a new broad spectral band is observed which is deeper than the original bound exciton $\left(I_{2}\right)$ line. In contrast with previously known centers, this band shows remarkably large shift as a function of light intensity, which can be described by scaling laws. A theoretical model is proposed, which considers the excitonic emission in porous media. According to this model, the coulombic energy of the exciton increases due to the reduced polarizability of the composite media. Good agreement is obtained between theory and experimental data, and self-consistency is established for the parameters of the theory.
\end{abstract}

\section{Introduction}

CdS belongs to the II-VI family of compounds with a wide range of applications. The photoluminescence of CdS has been investigated in detail in the past ${ }^{1-5}$. Controlled photoelectrochemical etching (PE) of CdS (as well as other II-VI materials) leads to an appreciable improvement in its photovoltaic properties ${ }^{6-8}$. A porous (fractal type) morphology is obtained by this surface treatment, which leads to the coupling of light in oblique angles and hence to a reduced reflectivity ${ }^{9}$. It was indicated that PE of semiconductors leads to a preferential etching of shallow donor impurities from a layer near the semiconductor surface $e^{10-12}$. Low temperature photoluminescence is used in this work to investigate the optical properties of the porous layer. The PL spectra of these surfaces are dominated by a new kind of transition. To discuss the origin of this transition, a theory is developed, which attribute the shift of the peak to increasing of electron hole interaction in the porous media. The theory yields quantitative agreement with experimental results and self consistency among the parameters.

\section{Experiment}

Low resistivity n-type CdS(CdSe) crystals were polished, chemically etched, and finally photoetched in acidic solution, under anodic bias ${ }^{6,8}$. A standard low temperature PL set-up was employed for the measurements.

* Present address: Department of Chemistry and Solid State Institute, Technion, Israel Institute of Technology, Haifa 32000, Israel.
Using scanning electronmicroscope (SEM), a porous fractal-type morphology is revealed after PE. A dense pattern of etch-pits ranging in size between $100-2000 \AA$ could be resolved (inset of Fig.1). Although smaller etch pits down to a single vacancy are likely to exist, they could not be resolved with the present microscope. The sponge like layer extends to about 1-2 $\mu$, beneath the surface.

The salient features in the low temperature photoluminescence spectrum of a chemically etched n-type CdS are: donor bound exciton $\left(I_{2}\right)$ at $486.4 \mathrm{~nm}$, and its

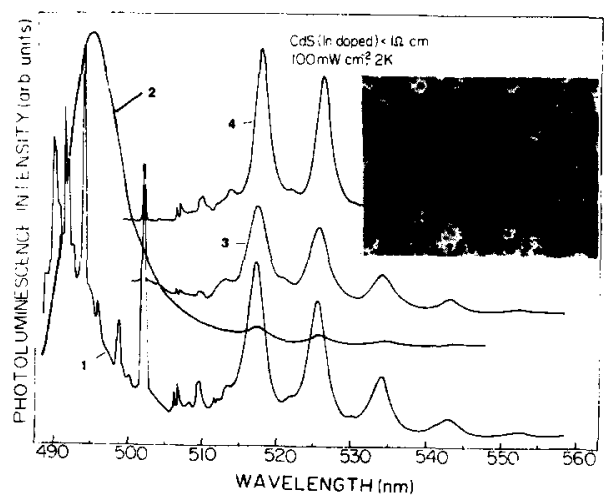

Fig.1 Low temperature photoluminescence of CdS: 1 . $\mathrm{HCl}$ etched $\left(\lambda_{e x}=458 \mathrm{~nm}\right) ; 2$. porous-PE $\left(\lambda_{e x}=458 \mathrm{~nm}\right)$; 3. porous-PE $\left(\lambda_{e x}=488 \mathrm{~nm}\right)$; 4. $\mathrm{HCl}$ etched $\left(\lambda_{e x}=488 \mathrm{~nm}\right)$. The inset shows an SEM image of the porous (PE) CdS surface. 
LO replica; a free to bound transition at $513.8 \mathrm{~nm}$; a donor-acceptor pair luminescence $(517 \mathrm{~nm})$, and its LO replica (curve 1 in Fig.1). This spectra agrees with the published data ${ }^{1-5}$.

The spectrum of the PE sample, however, is dominated by wide asymmetric emission (curve 2 in Fig.1). Its intensity and position is strongly influenced by the light intensity. Under weak light intensity (ca. $1 \mathrm{~mW} / \mathrm{cm}^{2}$ ) the peak position is close to that of the DAP. Under stronger light intensity ( $>100 \mathrm{~mW} / \mathrm{cm}^{2}$ ) this band becomes strong and centered close to the bulk exciton energy. On increasing the temperature this emission slowly fades out and the free to bound transition predominates, as for the bulk (chemically etched) material. If sub bandgap light (488nm) is used for excitation, the usual bulk luminescence is observed (curves 3 and 4 in Fig. 1). Very similar spectra were obtained for PE CdSe.

The improved photovoltaic (photoresponse) of the sample suggests that fewer impurity atoms are left in the porous layer after $\mathrm{PE}^{10-12}$. This is substantiated by the fact that all the emission lines associated with donor atoms are suppressed (curve 2 in Fig.1). The position of an exciton line is insensitive to the intensity of the excitation light in the $1-5000 \mathrm{~mW} / \mathrm{cm}^{2}$ range. Typically the maximum of the DAP luminescence varies by $1 \mathrm{meV}$ for an order of magnitude change in the light intensity ${ }^{13}$. Notwithstanding, the emission band of the PE surface changes by more than $70 \mathrm{meV}$ in this range. Such a remarkable shift must be associated with a strong variation in the local electronic properties near the emitting center. Therefore it is not likely that the new band in the PL spectrum can be associated with a known excitonic transition.

A plot of the shift of the peak maximum (relative to the bulk exciton energy) $-\Delta_{m}$, the value of luminescence intensity at the maximum $-I_{m}$, and the integral intensity of the peak $-P$, against the intensity of excitation light$W_{0}$ in a double logarithmic scale, shows that these plots can be represented as power dependencies (Fig.2, inset).

$$
\begin{gathered}
\Delta_{m} \propto W_{0}^{-\eta_{\Delta}}, \quad I_{m} \propto W_{0}^{\eta_{I}}, \quad P \propto W_{0}^{\eta_{P}} \\
\eta_{\Delta}=.115 \mp .01, \quad \eta_{I}=1.15 \mp .15, \quad \eta_{P}=1.06 \mp .13
\end{gathered}
$$

Moreover, a plot of the normalized luminescence intensity $I(\Delta) / I_{m}$ against frequency shift $\Delta / \Delta_{m}$ can be represented by a universal function for all intensities in the range from $.06 \mathrm{~mW} / \mathrm{cm}^{2}$ to $600 \mathrm{~mW}^{2} / \mathrm{cm}^{2}$. The change in the position of the maximum with light intensity suggests that kinetics is involved in the luminescence process. Additionally, the superlinear behavior of $I_{m} / W_{0}$ and $P / W_{0}$ with $W_{0}$ is an evidence that the kinetics is not linear. Any theoretical explanation has to take these experimental facts into consideration.

The important indication for the nature of this phenomena is the value of the shift and width of the luminescence peak. The shift of the luminescence peak is larger a few times than the exciton binding energy (39meV for bulk CdS, $20 \mathrm{meV}$ for CdSe)

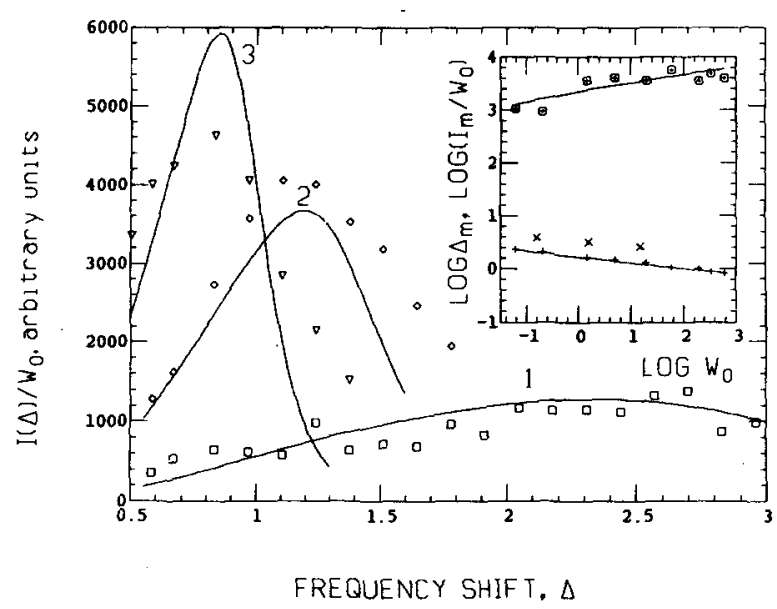

Fig.2 Scaled photoluminescence intensity of the porous CdS vs. scaled energy shift of the exciton energy. Different symbols denotes different incident light intensities $W_{0} . \overline{-}-0.06 \mathrm{~mW} / \mathrm{cm}^{2}$, 。 $-20 \mathrm{~mW} / \mathrm{cm}^{2}, \quad \nabla-600 \mathrm{~mW} / \mathrm{cm}^{2}$. Solid lines are theoretical curves given by Eq.(12),(13) for the same light intensities: 1 - for $0.06 \mathrm{~mW} / \mathrm{cm}^{2}$, 2 -for $20 \mathrm{~mW} / \mathrm{cm}^{2}$, 3-for $600 \mathrm{~mW} / \mathrm{cm}^{2}$ Parameters: saturation level $W_{\mathrm{a} 1}=370 \mathrm{~mW} / \mathrm{cm}^{2}, I_{0}=$ $2.6 \times 10^{6} \mathrm{sec}^{-1}, i=1.3$ In the inset, the validity of scaling given by Eqs.1 vs. incident light intensity of two experimental parameters: + - shift of the luminescence peak $\left(\Delta_{m}\right)$ for $C d S, \times$ - for a $C d S e ; \oplus-$ maximum of the luminescence intensity $\left(I_{m} / W_{0}\right)$, is shown. Solid lines are theoretical curves given by Eqs. (12),(19).

\section{Theory}

To give a theoretical description for the broad luminescence peak of PE CdS (CdSe) and its dependence on light intensity it is assumed that recombination of excitons occurs within the porous media. The dielectric constant of the pores $\left(\epsilon_{p}=1\right)$ is smaller than that of the bulk semiconductor $\left(\epsilon_{b u l k} \approx 10\right)$. Consequently the coulombic interaction between electron and hole increases accordingly ${ }^{14,15}$. Quantum localization of excitons within the pores, similar to that observed in disordered semiconductor alloys ${ }^{16-18}$, leads to a larger effective mass. However this effect is likely to have a smaller influence on the exciton energy in porous media and is therefore disregarded ${ }^{19}$. The exciton energy $E$ depends on exciton position $\vec{r}$ in between the pores as well as on the pores' configuration. Consequently $E(\vec{r})$ is distributed in a broad range of energies, starting from a bulk value $E_{\text {bulk }}$ up to some maximum value $E_{\text {max }}$, which corresponds to the exciton which is confined near the largest concentration of pores. The normalized shift of the exciton energy (relative to the binding energy in the bulk) is: 


$$
\begin{gathered}
\Delta(\vec{r})=\frac{E(\vec{r})}{E_{b u l k}}-1=\frac{\Delta \omega(\vec{r})}{\omega_{g}-\omega_{b u l k}}=\frac{\omega_{b u l k}-\omega(\vec{r})}{\omega_{g}-\omega_{b u l k}}, \\
E_{b u l k}=E_{g}-E_{I_{2}}, \quad \omega_{g}=E_{g} / \hbar, \quad \omega_{b u l k}=E_{I_{2}} / \hbar .
\end{gathered}
$$

Here $E_{g}$ is the energy gap, and $E_{I_{2}}$ is the luminescence energy of the donor bound exciton. by

Provided $\Delta(\vec{r})$ is known the line shape $I(\Delta)$ is given

$$
I(\Delta)=\oint_{\Delta(\vec{r})=\Delta} J(u, v, \Delta) \quad \frac{\partial(x, y, z)}{\partial(u, v, \Delta)} d u d v .
$$

Here $u$ and $v$ are arbitrary variables on a surface of constant energy shift $\Delta(\vec{r})=\Delta, \quad \Delta$ is a coordinate normal to this surface., The coordinates $u, v$ and $\Delta$ substitute the cartesian coordinates $x, y, z . J(\vec{r})$ is the luminescence intensity under certain light intensity $W(\vec{r})$.

To find the function $J(u, v, \Delta)$ one has to know the luminescence kinetics. For a kinetic law of order $i=1,2$ 21 one obtains:

$$
\begin{gathered}
J(\vec{r})=A(\vec{r}) \frac{W^{i}(\vec{r}) / W_{s}^{i}(\vec{r})}{1+W^{i}(\vec{r}) / W_{s}^{i}(\vec{r})} \\
A(\vec{r})=n(\vec{r}) / \tau_{l}
\end{gathered}
$$

Here the functions $A(\vec{r})$ and the 'saturation' intensity $W_{s}(\vec{r})$ are combinations of material parameters, such as: density of luminescence centers- $n$; luminescence time $\tau_{l}$; lifetime of electron-hole pair (free exciton); generation rate for a given exciting light flux $\mathrm{W}$, and so on. Generally these parameters and consequently $A$ and $W_{s}$ are functions of $\vec{r}$ due to the inhomogeneity of the porous media.

In the bulk media both wavelength and light absorption length $l_{b u l k}$ are $\sim .1 \mu$. The light propagation through such media is a very complicated process. It includes such processes as strong refraction and absorption by a system of channels of different lengths up to a few microns, and thickness in the range from $.5 \mu$ and at least down to $100 \AA$. Light may penetrate inside the media much deeper than $l_{\text {bulk }}$ through the larger channels (with a diameter larger than $\sim .1 \mu$ ). As a result the function $W(\vec{r})$ is very complicated. The average intensity is assumed to decay exponentially with $z$ which is the distance to the surface of the large channels

$$
W(\vec{r})=c W_{0} \exp (-z / l), \quad c<1
$$

Here $W_{0}$ is the intensity of the incident light and $l$ is a characteristic decay length, which is determined both surface, since in the process of photoetching most impurities were etched out ${ }^{10-12}$. As a result the concentration of luminescence centers decays exponentially from the boundary of the porous layer $(\sim L)$ to the surface.

$$
n(\vec{r})=n_{0} \exp \left(z / l_{1}\right), \quad z<L
$$

In this case Eqs.(4)-(11) yield a simple lineshape:

$$
\begin{gathered}
I(\Delta)=A_{0} \frac{w_{1}^{i} f^{i a}(\Delta)}{1+w_{1}^{i} f^{i a}(\Delta)} f^{-b}(\Delta) \varphi(\Delta), \\
f(\Delta)=\frac{3.414 \Delta}{\sqrt{1+\Delta}(1+\sqrt{1+\Delta})}, \varphi(\Delta)=\frac{(1+\sqrt{1+\Delta})}{\Delta(1+\Delta)} \\
a=L / l, \quad b=L / l_{1}, \quad w_{1}=W_{0} / W_{s 1}, \\
f(1)=1, \quad W_{s 1}=W_{s}\left(3 X_{0} / 2\right)^{a}
\end{gathered}
$$

Fitting the experimental lineshape (see Fig.2) by using the expression (9) it is possible to obtain the parameter $W_{s 1}$ and the parameters $i, a$ and $b ;$

$$
W_{s 1}=370 \mathrm{~mW} / \mathrm{cm}^{2}, \quad i=1.30 \mp .15, \quad a \approx b \approx 16 \mp 2 .
$$

The value $l=L / a \approx .1 \mu$ is a reasonable estimate for the effective penetration length of the incident light. The value $l_{1}=L / b \approx .1 \mu$ shows that the ratio of density of impurities on the surface $n(0)$ to density impurities in bulk $n(L)$ is a small value $\sim 10^{-5}-10^{-6}$ (due to the process of photoetching). This value coincides with the estimate based on saturation light intensity $W_{s 1}$ (see Discussion).

Solid lines in Fig.2 demonstrate that the 'small pore' model satisfactorily explains the experimental data. However, SEM observations revealed mainly 'large' pores, hence it would be natural to assume that large pore theory would be a better approach. ${ }^{25}$

\section{Discussion}

To explain the dependence of luminescence line shape on intensity of the exciting light we assume that the luminescence centers are distributed inside the porous structure and that the number of luminescence centers decreases extremely quickly with $\Delta$. The density of centers with $\Delta=2.5$ is $\approx 10^{6}-10^{7}$ times less than density of centers with $\Delta=0.5$. Nevertheless, under a low light intensity they trap most of the pairs. In the small pore case this is achieved by the strong absorption of light near the surface and total correlation between light intensity and $\Delta$. It is possible to estimate the density of luminescence centers, $n_{0}(\Delta)$, with $\Delta$ in a certain interval of frequencies for any given value of $W_{a}$. Most of the light is absorbed in a layer of thickness $2 l \sim .2 \mu$. In accordance with equation (8) the luminescence centers in this layer have frequency shifts within the interval $2.3<\Delta<3.0$ (tail of peak un$\operatorname{der} W_{0}=.06 \mathrm{~mW} / \mathrm{cm}^{2}$ ) (Fig.2). Saturation means that their density $\sim n_{0}$ should be much less than the flux of photons per unit volume for a time $\tau_{l} \sim 10^{-8}-10^{-9} s$ :

$$
n_{0}<10^{14} \times W_{0} \times \tau_{l} / l \approx 10^{10} \mathrm{~cm}^{-3}
$$

The value of $n_{0}$ is at least 6 orders of magnitude smaller than the bulk concentration of impurities. This fact corroborates suggestion that the semiconductor is cleaned of shallow donors in the process of photoetching.

To test our theory some additional experiments would be useful. The most direct approach would be to fill the pores with materials of larger dielectric con- 
stant. This procedure should shift all peaks to the bulk exciton peak. To obtain the distribution of luminescence centers as a function of distance from the surface it would be useful to remove thin surface layers in succession. However, to perform these modifications and to keep the porous structure intact is a complicated problem. More realistic is to investigate the dependence of lineshape on wavelength of exciting light. The value of penetration length $l_{b u l k}$ and consequently $l$ is a strong function of the excitation energy above bandgap. Thus it is possible to change essentially the main parameter $a=L / l$ of small pore theory. Alternatively, it is possible to change the wavelength of light in the process of photoetching and thus to change the thickness of the porous layer $L$. Preliminary experiments according to these lines were performed in the our laboratory. These experiments and the kinetics for the case of large pores will be reported elsewhere.

\section{References}

1. D.G. Thomas and J.J. Hopfieled, Phys. Rev., 116, 573 (1959); 128, 2135 (1962).

2. K. Colbow, Phys. Rev., 141, 742 (1966).

3. C.H. Henry, R.A. Faulkner, and K. Nassau, Phys. Rev., 183, 798 (1969).

4. M. Moroz, Y. Breda, and A. Honig, Solid State Commun., 47, 115 (1983).

5. R.T. Cox and J.J. Davies, Phys. Rev. B, 34, 8591 (1986).

6. R. Tenne and G. Hodes, Appl. Phys. Lett., 37, 428 (1980).

7. A. Kirch-DE Mesmaeker, A.M. Decoster, and J.Nasielski, Solar Energy Mater., 4, 203 (1981).

8. G. Hodes, D. Cahen, and H.J. Leamy, J. Appl. Phys, 54, 4676 (1983).

9. M.A.Green,"High Efficiency Silicon Solar Cells" Trans. Tech. Publications, Switzerland (1987).

10. R. Tenne, H. Flaisher, and R. Triboulet, Phys. Rev. $B, 29,5799$ (1984).

11. R. Garuthara, M. Tomkiewicz, and R. Tenne, Phys. Rev. B, 31, 7844 (1985).

12. R. Tenne, H. Mariette, C. Levy-Clement, and R. Jager-Waldau, Phys. Rev. B, 36, 1204 (1987).
13. G.F. Neumark, S.P. Herko, and B.J.Fitzpatrick, 13th Intl. Conf. Defects in Semiconductors, eds. L.C. Kimerling and J.M. Parsey, 14a, 1205 (1984).

14. A.V. Chaplik, and M.V. Entin, Sov. Phys. JETP., 34, 1335, (1972)

15. L.V. Keldysh, JETP Lett., 29, 658 (1979).

16. S. Permagorov, A. Raznitskii, S. Verbin, G.O. Müller, P. Flögel and M.Nikiforova, Phys. Stat. Sol. $B, 113,589$ (1982).

17. E. Cohen and M.D. Sturge, Phys. Rev. B, 25, 3828 (1982).

18. D. Lee, A. Mysyrovicz, A.V. Nurmikko and B.J. Fitzpatrick, Phys. Rev. Lett., 58, 1475, (1987)

19. Up to porosity smaller than $65 \%$ no localization phenomena was observed in the porous $S_{i}^{20}$.

20. A. Halimaoui, C. Oules, G.Bomchil, A.Bsiesy, F.Gaspard, R.Herino, M. Ligeon, and F.Muller Appl. Phys. Lett., 59, 304, (1991)

21. The fractional value $i \approx 1.30$, which is consistent with the experimental data, is an evidence that the light induced electrons and holes do not always diffuse in pairs to the luminescence centers, i.e. are correlated less than in the bulk ${ }^{23}$ Possible explanation is that strong electric fields near internal surfaces increase the rate of decay of the free exciton into an electron and a hole.

22. I.Webman, J. Jortner, M.N. Cohen, Phys. Rev. $B, 15,5712$ (1977).

23. Amorphous Semiconductors, Topics in Applied Physics, 36 ed. M.H. Brodsky, Springer-Verlag Berlin, Heidelberg, New-York, 1979 p.110, G.A.N. Connel.

24. R.J. Temkin, W.Paul, G.A.N. Connel, Adv. Phys., 22, 581 (1973).

25. Eqs.(12),(13) are not of 'scaling' type. Nevertheless, as it is shown in Fig.2 (inset), they imitate 'scaling' dependencies. The reason is that in the interval $1<\Delta<2.5$ both $f(\Delta)$ and $\varphi(\Delta)$ may be approximated by power functions with a satisfactory accuracy. It is possible that experimental 'scaling' dependencies are approximate. Alternatively, these equations are reflection of some intrinsic property of porous media. At any case these dependencies has to break close to the bulk exciton peak. 